\title{
Weak chaos and metastability in a symplectic system of many long-range-coupled standard maps
}

\author{
Luis G. Moyand* \\ Centro Brasileiro de Pesquisas Físicas \\ Rua Xavier Sigaud 150, \\ 22290-180 Rio de Janeiro-RJ, Brazil \\ Ana P. Majtey \\ Facultad de Matemática, Astronomía y Física, \\ Universidad Nacional de Córdoba \\ Ciudad Universitaria, 5000, Córdoba, Argentina \\ CONICET \\ Constantino Tsalli: \\ Santa Fe Institute \\ 1399 Hyde Park Road, Santa Fe, NM 87501, USA \\ and \\ Centro Brasileiro de Pesquisas Físicas \\ Rua Xavier Sigaud 150, \\ 22290-180 Rio de Janeiro-RJ, Brazil
}

(Dated: June 10, 2018)

\begin{abstract}
We introduce, and numerically study, a system of $N$ symplectically and globally coupled standard maps localized in a $d=1$ lattice array. The global coupling is modulated through a factor $r^{-\alpha}$, being $r$ the distance between maps. Thus, interactions are long-range (nonintegrable) when $0 \leq \alpha \leq 1$, and short-range (integrable) when $\alpha>1$. We verify that the largest Lyapunov exponent $\lambda_{M}$ scales as $\lambda_{M} \propto N^{-\kappa(\alpha)}$, where $\kappa(\alpha)$ is positive when interactions are long-range, yielding weak chaos in the thermodynamic limit $N \rightarrow \infty$ (hence $\lambda_{M} \rightarrow 0$ ). In the short-range case, $\kappa(\alpha)$ appears to vanish, and the behaviour corresponds to strong chaos. We show that, for certain values of the control parameters of the system, long-lasting metastable states can be present. Their duration $t_{c}$ scales as $t_{c} \propto N^{\beta(\alpha)}$, where $\beta(\alpha)$ appears to be numerically consistent with the following behavior: $\beta>0$ for $0 \leq \alpha<1$, and zero for $\alpha \geq 1$. All these results exhibit major conjectures formulated within nonextensive statistical mechanics (NSM). Moreover, they exhibit strong similarity between the present discrete-time system, and the $\alpha$-XY Hamiltonian ferromagnetic model, also studied in the frame of NSM.

PACS numbers: 05.20.-y, 05.45.-a, 05.70.Ln, 05.90.+m

Keywords: Nonlinear dynamics, Weak chaos, Metastable (quasistationary) states, Nonextensive statistical mechanics
\end{abstract}

\section{INTRODUCTION}

The study of complex systems has been lately one the most active areas of investigation. This multidisciplinary subject has interest in natural sciences as well as in social and artificial systems. In physics, a major area involved is statistical mechanics. It is precisely for such systems that nonextensive statistical mechanics (NSM) 1] appears to have its applicability. Typical features present in complex systems are long-range interactions, long-term memory, fractal phase-space structure, scale-free network structure, or even combinations of these characteristics. Quite frequently these systems cannot be correctly described by the well-established Boltzmann-Gibbs statis-

\footnotetext{
*e-mail address: moyano@cbpf.br

†e-mail address: amajtey@famaf.unc.edu.ar

‡e-mail address: tsallis@santafe.edu
}

tical mechanics (BGSM). Indeed, they often fail to verify its basic assumptions (equiprobability of phase-space occupation 2 2 and ergodicity 3 at the thermal equilibrium state). NSM generalises the usual BGSM formalism through the definition of the q-entropy $S_{q} \equiv \frac{1-\int d x[p(x)]^{q}}{q-1}$ (with $S_{1}=S_{B G} \equiv-\int d x p(x) \ln p(x)$, where $B G$ stands for Boltzmann-Gibbs) under simple constraints [4].

This theory has been extensively applied to nonlinear dynamical systems [1, 5, 6, 7], as they are one of the most widespread and useful ways for modelling complex phenomena. For example, Hamiltonian systems are at the core of physics and consequently their relevance is evident. There has been an important number of results indicating that certain conservative models (e.g., the $\alpha$ XY model 8, 9], the $\alpha$-Heisenberg model 10, 11], the $\alpha$-Lennard-Jones-like gas [12]) can present a behaviour departing from the one predicted by the BG formalism. For certain classes of initial conditions and parameters, the system is prevented from virtually attaining its ex- 
pected equilibrium state in finite time when $N \rightarrow \infty$. In other words, if the $N \rightarrow \infty$ limit is taken before the $t \rightarrow \infty$ limit, the system becomes nonergodic. This nonergodicity is also reflected in a variety of anomalous behaviours such as non-Gaussian momenta probability density functions, negative specific heat, aging, and others 13, 14, 15, 16]. On the other hand, other (simpler) nonlinear dynamical systems, as for example maps, have emerged in many contexts, very often exhibiting new and interesting results. Among these systems we find the logistic map, the standard map, coupled map lattices, discretised Lorentz gas, and many others [17]. Both low- and high-dimensional discrete-time dynamical systems have been studied under the framework of NSM, exhibiting various connections with Hamiltonian dynamics [18, 19, 20].

It is known quite well that the number of degrees of freedom of a system defines the possibilities that its dynamics may approach. A clear illustration of this fact in low-dimensional systems (e.g., the 2-dimensional Chirikov-Taylor standard map) is the KAM tori, a complex structure in the map phase-space that separates chaotic from regular regions. While much is known for systems with few degrees of freedom, the situation is more intricate when many elements are involved. This is because the $\Gamma$ phase-space grows in an extremely rapid manner. In addition to this, studying continuous-time many-body systems can turn out to be very difficult, if not impossible at all, due to the considerable computational time needed to integrate the evolution equations. This is even more so when long-range forces are involved, as it is not justified to neglect any interaction between the elements.

An alternative for this problem is the substitution of the continuous-time system for simpler discrete-time systems, such as maps, which conserve many of the important features of the physics involved. This substitution is in fact justified interpreting a dimensional map system as the intersection (in the Gibbs $\Gamma$ phase-space) of a Poincaré plane with the orbit of a higher dimensional Hamiltonian. More precisely, if we consider a time-independent Hamiltonian system with $(N+1)$ degrees of freedom $((2 N+2)$-dimensional $\Gamma$ phase-space $)$, a $(2 N)$-dimensional symplectic map is the result of taking a Poincaré section over the constant energy hypersurface [17. The recurrence time is discrete and the map is useful in displaying various properties of the original Hamiltonian system.

NSM concepts have been shown to have an important role in low-dimensional maps at the edge of chaos. The archetypical logistic map (a $D=1$, dissipative, nonlinear map) is one of the most widely studied dissipative systems. In part due to its simplicity, it is often used to illustrate many of the most important features of chaos. In recent works, Robledo and Baldovin 21] have analytically proved, using standard renormalisation-group techniques, that the dynamics of the logistic map at its critical point is unmistakenly well-described within a NSM frame. The sensitivity to initial conditions is a $q$-exponential function [22], and is related to the entropy production through the $q$-generalised Pesin-like identity, linking the sensitivity to initial conditions to the $q$-entropy $S_{q}$ with $q=0.2445 \ldots$ Moreover, the logistic-map with noise (a Langevin-like generalisation of the usual logistic map) has been found to present two-step relaxation processes and aging (presenting interesting common points with slow glassy dynamics 23 ]). Other aspects of the NSM formalism were also studied in the more general case of one-dimensional $z$-logistic family of maps considering the attractor fractal nature [24]. Regarding two-dimensional $(D=2)$ dissipative systems (as the Henón map or its linearised version, the Lozi map), results indicate that it presents the same value of $q$ as the logistic map, therefore suggesting a common universality class [25]. Regarding two-dimensional $(D=2)$ conservative maps, a very interesting example, the triangle Casati-Prosen map (mixing, ergodic, but with vanishing Lyapunov exponent), has been recently been studied in connection to the entropy $S_{q}$ with $q=0$ [26].

Moving further into conservative discrete-time systems, two-step relaxation has been also observed at the edge of chaos for the Chirikov-Taylor standard map, a paradigmatic one for 2-dimensional symplectic maps. This map has been thoroughly studied and explained by means of the KAM-theorem [17]. This map is known to be area preserving, hence its variables are often considered as "coordinate" and "momentum", in analogy with Hamiltonian systems. Along this lines, it is appealing to look to the more general case of a symplectic system of $N$ coupled standard maps $(D=2 N, N \geq 1)$. Different efforts were done for values of $N$ as low as $N=2$ up to $N=500$ 20, 27, 28]. In these cases, the same two-step relaxation was found as well as other features pointing to a NSM applicability. But in all these cases, the coupling was done trough the momentum variables. Even though this has its own interest, it would be instructive to see the effects of coupling such a system through the coordinate variables, as in more realistic situations.

In this work we will study a high-dimensional globally coupled conservative map system that, as discussed above, presents many of the characteristics of Hamiltonian dynamics. Our purpose is to contribute to the understanding of the role that NSM plays in the anomalous features present in long-range-interacting dynamical systems. Our results show interesting similarities between this map model and many-body long-range-interacting Hamiltonian dynamics, in particular the $\alpha$-XY model. In both cases, long-lasting metastable states, as well as weak chaos, are present in the thermodynamic limit. Both features point out that under certain initial conditions, orbits do not visit with equal probability the entire $\Gamma$ phase space, or in other words there is a failure in ergodicity.

In the next section we introduce and describe the model, as well as some relevant details of our simulations. In section ПII we present our numerical results. We report on the scaling behaviour of the largest Lyapunov expo- 
nent with the system size $N$, and point out connections with Hamiltonian dynamics. We analyse the relaxation to equilibrium, and we make a systematic characterisation as a function of the system parameters. Finally, a discussion and summary are presented in section IV

\section{MODEL}

Our model is a set of $N$ symplectically coupled (hence conservative) standard maps, where the coupling is made through the coordinates as follows:

$$
\begin{aligned}
& \theta_{i}(t+1)=\theta_{i}(t)+p_{i}(t+1) \quad(\bmod 1), \\
& p_{i}(t+1)=p_{i}(t)+\frac{a}{2 \pi} \sin \left[2 \pi \theta_{i}(t)\right]+ \\
& \frac{b}{2 \pi \hat{N}} \sum_{\substack{j=1 \\
j \neq i}}^{N} \frac{\sin \left[2 \pi\left(\theta_{i}(t)-\theta_{j}(t)\right)\right]}{r_{i j}^{\alpha}}(\bmod 1)
\end{aligned}
$$

where $t$ is the discrete time $t=1,2, \ldots$, and $\alpha \geq 0$. The $a$ parameter is the usual nonlinear constant of the individual standard map, whereas the $b$ parameter modulates the overall strength of the long-range coupling. Both parameters contribute to the nonlinearity of the system; it becomes integrable when $a=b=0$. For simplicity, we have studied only the cases $a>0, b>0$, but we expect similar results when one or both of these parameters are negative. The systematic study of the whole parameter space is certainly welcome. Notice that, in order to describe a system whose phase space is bounded, we are considering, as usual, only the torus (mod 1). Additionally, the maps are localised in a one dimensional $(d=1)$ regular lattice with periodic boundary conditions. The distance $r_{i j}$ is the minimum distance between maps $i$ and $j$, hence it can take values from unity to $\frac{N}{2}\left(\frac{N-1}{2}\right)$ for even (odd) number $N$ of maps. Note that $r_{i j}$ is a fixed quantity that, modulated with the power $\alpha$, enters Eq. (1) as an effective time-independent coupling constant. As a consequence, $\alpha$ regulates the range of the interaction between maps. The sum is global (i.e., it includes every pair of maps), so the limiting cases $\alpha=0$ and $\alpha=\infty$ correspond respectively to infinitely long range and nearest neighbours. In our case $d=1$, thus $0 \leq \alpha \leq 1(\alpha>1)$ means long-range (short-range) coupling. Moreover, the coupling term is normalised by the $\operatorname{sum}[9,29] \tilde{N} \equiv d \int_{1}^{N^{1 / d}} d r r^{d-1} r^{-\alpha}=\frac{N^{1-\alpha / d}-\alpha / d}{1-\alpha / d}$, to yield a non-diverging quantity as the system size grows (for simplicity, we have replaced here the exact discrete sum over integer $r$ by its continuous approximation).

If $G(\bar{x})$ denotes a map system, then $G$ is symplectic when its Jacobian $\partial G / \partial \bar{x}$ satisfies the relation [17]:

$$
\left(\frac{\partial G}{\partial \bar{x}}\right)^{T} J\left(\frac{\partial G}{\partial \bar{x}}\right)=J
$$

where the superindex $T$ indicates the transposed matrix, and $J$ is the Poisson matrix, defined by

$$
J \equiv\left(\begin{array}{cc}
0 & I \\
-I & 0
\end{array}\right)
$$

$I$ being the $N \times N$ identity matrix. A consequence of Eq. (2) is that the Jacobian determinant $|\partial G / \partial \bar{x}|=1$, indicating that the map $G$ is (hyper)volume-preserving. In particular, for our model

$$
\frac{\partial G}{\partial \bar{x}}=\left(\begin{array}{cc}
I & I \\
B & (I+B)
\end{array}\right)
$$

where $\bar{x}$ is the $2 N$-dimensional vector $\bar{x} \equiv(\bar{p}, \bar{\theta})$, and

$$
B=\left(\begin{array}{cccc}
K_{\theta_{1}} & c_{21} & \ldots & c_{N 1} \\
c_{12} & K_{\theta_{2}} & \ldots & c_{N 2} \\
\vdots & \vdots & \vdots & \vdots \\
c_{1 N} & c_{2 N} & \ldots & K_{\theta_{N}}
\end{array}\right)
$$

with

$$
K_{\theta_{i}}=a \cos \left[2 \pi \theta_{i}(t)\right]+\frac{b}{\tilde{N}} \sum_{j \neq i} \frac{\cos \left[2 \pi\left(\theta_{i}(t)-\theta_{j}(t)\right)\right]}{r_{i j}^{\alpha}},
$$

and

$$
c_{i j}=c_{j i}=-\frac{b}{\tilde{N}} \frac{\cos \left[2 \pi\left(\theta_{i}(t)-\theta_{j}(t)\right)\right]}{r_{i j}^{\alpha}},
$$

where $i, j=1, \ldots, N$. It can be seen that,

$$
\left(\frac{\partial G}{\partial \bar{x}}\right)^{T}=\left(\begin{array}{cc}
I & B \\
I & (I+B)
\end{array}\right)
$$

hence

$$
\left(\frac{\partial G}{\partial \bar{x}}\right)^{T} J=\left(\begin{array}{cc}
-B & I \\
-(I+B) & I
\end{array}\right)
$$

This quantity, multiplied (from the right side) by the matrix (4) yields $J$. Therefore our system is symplectic. Consequently, the $2 N$ Lyapunov exponents $\lambda_{1} \equiv$ $\lambda_{M}, \lambda_{2}, \lambda_{3}, \ldots, \lambda_{2 N}$ are coupled two by two as follows: $\lambda_{1}=$ $-\lambda_{2 N} \geq \lambda_{2}=-\lambda_{2 N-1} \geq \ldots \geq \lambda_{N}=-\lambda_{N+1} \geq 0$. In other words, as a function of time, an infinitely small length typically diverges as $e^{\lambda_{1} t}$, an infinitely small area diverges as $e^{\left(\lambda_{1}+\lambda_{2}\right) t}$, an infinitely small volume diverges as $e^{\left(\lambda_{1}+\lambda_{2}+\lambda_{3}\right) t}$, an infinitely small $N$-dimensional $h y$ pervolume diverges as $e^{\left(\sum_{i=1}^{N} \lambda_{i}\right) t}\left(\sum_{i=1}^{N} \lambda_{i}\right.$ being in fact equal to the Kolmogorov-Sinai entropy rate, in agreement with the Pesin identity), an infinitely small $(N+1)$ hypervolume diverges as $e^{\left(\sum_{i=1}^{N-1} \lambda_{i}\right) t}$, and so on. For example, a $(2 N-1)$-hypervolume diverges as $e^{\lambda_{1} t}$, and finally a $2 N$-hypervolume remains constant, thus recovering the conservative nature of the system (of course, this corresponds to the Liouville theorem in classical Hamiltonian dynamics). 
For $\alpha=0$, similar models exist in the literature though in different contexts 19, 30, 31]. The present particular choice for the coupling was made with the purpose of comparison with many-body Hamiltonian systems. Indeed, one can derive the map set of equations (1) by applying a discretisation procedure to the $\alpha$-XY model with an external field (for more details see [17, 31]). As a consequence of having $N-1$ terms in the coupling summation and the fact that there are $N$ maps, the simulation times are of order $O\left(N^{2}\right)$. For this reason it is a difficult task to numerically simulate (11) for large values of $N$. To overcome this problem, we used an algorithm that takes advantage of the symmetry of the lattice 32 and shortens the simulation time to $O(N \ln N)$.

Initial coordinates and momenta were randomly taken from the following uniform distributions: $\theta_{i} \in\left[\theta_{0}-\right.$ $\left.\delta \theta, \theta_{0}+\delta \theta\right]$ and $p_{i} \in\left[p_{0}-\delta p, p_{0}+\delta p\right]$. For the coordinates we used $\theta_{0}=\delta \theta=0.5$ (i.e., $\theta_{i} \in[0,1]$, homogeneous coordinate initial conditions). For the momenta, we concentrated in two cases. To study the sensibility to initial conditions we used a uniform distribution over the whole phase-space $\left(p_{0}=0.5\right.$ and $\left.\delta p=0.5\right)$. In the analysis of the relaxation to equilibrium we used a thin waterbag intial condition with $p_{0}=0.3$ and $\delta p=0.05$. We also checked inhomogeneous initial conditions, in particular $p_{0}=\theta_{0}=0.3$ and $\delta p=\delta \theta=0.05$ (not shown in this paper). Note that in this case there is no traslational symmetry in the coordinates. For sufficiently long times, our simulations yield the same sensitivity to initial conditions that we obtain for the symmetric case $\theta_{i} \in[0,1]$. On the other hand, this inhomogeneous initial condition in the coordinates has an important influence in the shape of the relaxation to equilibrium. This will be further commented in the next section. We note that the systematic study of the role of initial conditions is very instructive, but it is out of the scope of the present work.

\section{RESULTS}

\section{A. Sensitivity to initial conditions}

In order to analyse the sensibility to initial conditions, we numerically studied the largest Lyapunov exponent (LLE) for different values of parameters $a, b, \alpha$ and $N$. We used the well-known method developed by Benettin et al 33]. As a consequence of the symplectic structure of (11), the Lyapunov spectrum in the $2 N$-dimensional phase space of the map is, as already discussed, characterised by $N$ pairs of Lyapunov coefficients, where each element of the pair is the negative of the other. Therefore, the LLE sets an upper bound for the absolute value of the entire spectrum of exponents.

We concentrated on the evolution of the LLE for different values of $N$ starting with $\theta_{0}=0.5, \delta \theta=0.5, p_{0}=0.5$ and $\delta p=0.5, \forall \alpha$. In a typical (sufficiently long) realisation, the finite-time LLE, $\lambda_{M}$, is a good estimator for the analytical definition of the LLE (both quantities will coincide when $t \rightarrow \infty$ [34]). We averaged between realisations (typically 100) in order to have small statistical fluctuations. We checked that, for appropriately long times, $\lambda_{M}$ does not depend on the initial conditions [35].

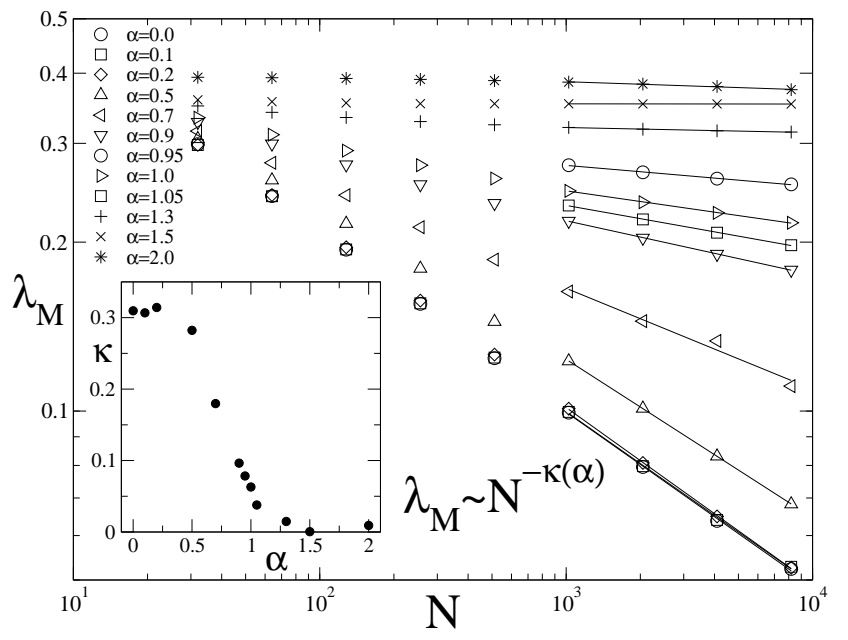

FIG. 1: Lyapunov exponent dependence on system size $N$ in $\log -\log$ plot, showing that $\lambda_{M} \sim N^{-\kappa(\alpha)}$. Initial conditions correspond to $\theta_{0}=0.5, \delta \theta=0.5, p_{0}=0.5$ and $\delta p=0.5$. Fixed parameters are $a=0.005$ and $b=2$. We averaged over 100 realisations. Inset: $\kappa$ vs. $\alpha$, exhibiting weak chaos in the limit $N \rightarrow \infty$ when $0 \leq \alpha \lesssim 1$.

In Fig. 1 1 we show the $N$-dependence of $\lambda_{M}$ for typical values of the interaction-range parameter $\alpha$, and fixed values of parameters $a=0.005, b=2$. Our results show that the value of $\lambda_{M}$ vanishes with increasing value of $N$ (consequently, so does the rest of the Lyapunov spectrum) as a power-law $\lambda_{M} \sim N^{-\kappa(\alpha)}$ for $\alpha \lesssim 1$, and is a positive constant for $\alpha>1(\kappa \approx 0)$. In the inset we detail $\kappa$ as a function of $\alpha$. This shows that the map system is weakly chaotic for long-range coupling $\left(\lambda_{M} \rightarrow 0\right.$ when $N \rightarrow \infty$ ), whereas for short-range interactions, $\lambda_{M}$ remains positive for all $N$, meaning strongly chaotic dynamics (as expected 36, 37]). Interestingly, this result is totally similar to the one numerically measured and analytically predicted for the $\alpha$-XY model [38], thus suggesting equivalent behaviours. Indeed, and as stated by Anteneodo and Vallejos, this scaling is typical of systems with couplings of the form $1 / r^{\alpha}[39]$. Preliminary simulations suggest that the fact that the weak chaos region extends slightly over $\alpha=1$ is an expected consequence of finite-size and finite-time effects.

The dependence of $\lambda_{M}$ with the nonlinear parameter $a$ for different ranges of the interaction $\alpha$ is shown in Fig. 2 We can see that, for $\alpha<1, \lambda_{M}$ decreases with $a$ and saturates for $a \ll 1$. This illustrates the influence of this nonlinear term. For increasing $a$, the sensibility to initial conditions raises. On the other hand $a$ has almost no effect when $\alpha>1$, where the $\lambda_{M}$ is approximately constant on the whole $a$-range. We verified that for $a>1$ a slight increase appears for $\alpha>1$ (as in the long-range 


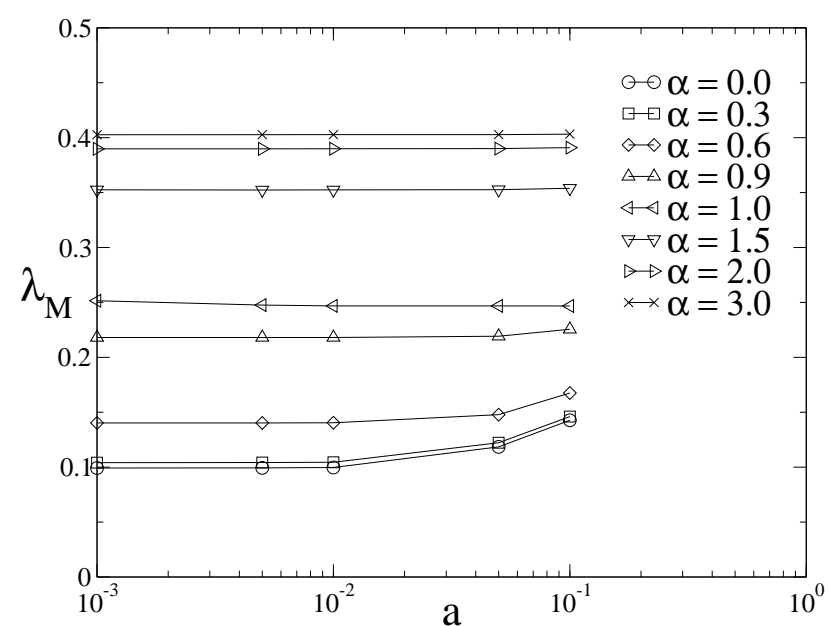

FIG. 2: Lyapunov exponent dependence on $a$ for different values of $\alpha$. Fixed constants are $N=1024$ and $b=2$. Initial conditions correspond to $\theta_{0}=0.5, \delta \theta=0.5, p_{0}=0.5$ and $\delta p=0.5$. We averaged over 100 realisations.

case), but this effect is negligible.

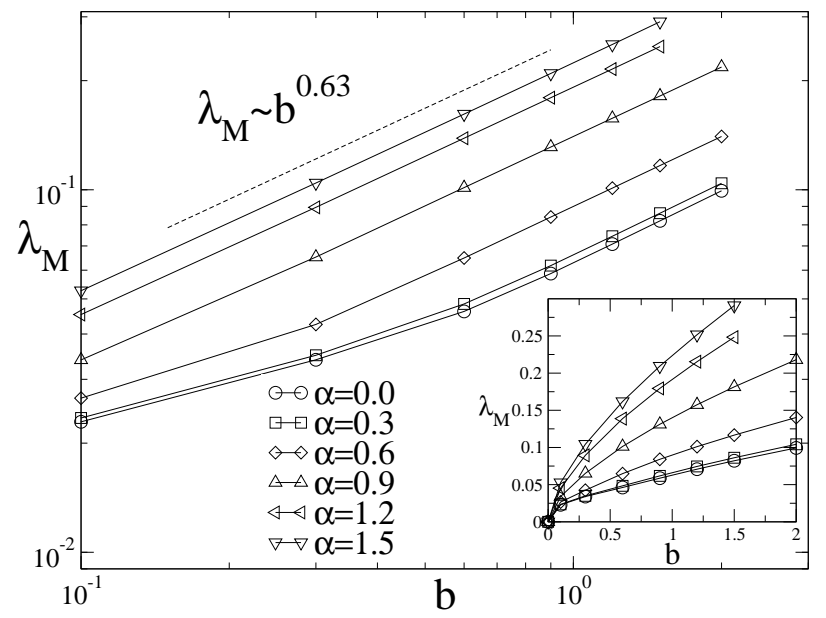

FIG. 3: Lyapunov exponent dependence on $b$ in log-log plot. Fixed constants are $N=1024$ and $a=0.005$. Initial conditions correspond to $\theta_{0}=0.5, \delta \theta=0.5, p_{0}=0.5$ and $\delta p=0.5$. We averaged over 100 realisations. Inset: Same data in linearlinear plot.

In Fig. 3 we exhibit the dependence on the coupling parameter $b$, also varying $\alpha$. For high values of $b(b \gg 1)$, $\lambda_{M}=c(\alpha) b^{0.63} \forall \alpha$, being $c(\alpha)$ a nonlinear function. Preliminary simulations show that this exponent varies only slightly with $a$ and $N$, suggesting that it may be unique. In the case $b=0$, we verified that $\lambda_{M}=0, \forall \alpha$. For $\alpha>1$ this scaling is valid even for $b \ll 1$. On the other hand, if $\alpha<1$, a deviation from this power-law behaviour emerges when $b \ll 1$.

Our characterisation illustrates the fact that, quite generically, the sensibility to initial conditions is strongly governed by the range of the interactions. Orbits are clearly chaotic in the short-range case (as expected) and strong numerical evidence of vanishing LLE emerges when interactions are long-range, revealing a weakly chaotic regime.

Our present results for the maximal Lyapunov exponent can be approximatively summarised as follows:

$$
\lambda_{M}(a, b, N, \alpha) \propto f_{\alpha}(b) N^{-\kappa(\alpha)},
$$

where $0 \leq a<<1 ; 0 \leq b \lesssim 2 ; N>>1, \alpha \geq 0$, and $f_{\alpha}(b)$ is some function of $(b, \alpha)$ (e.g., $f_{\alpha}(b) \propto b^{0.63}$ for $\left.\alpha>1\right)$.

\section{B. Temperature evolution}

As stated above, system (II) is symplectic, hence (hyper)volume preserving, like Hamiltonian systems. A consequence of this is that $\theta$ may be interpreted as a "coordinate" variable and $p$ as the conjugate "momentum". We may define a concept analogous to a temperature as twice the mean "kinetic energy" per particle [19, 28],

$$
T(t) \equiv \frac{1}{N} \sum_{i=1}^{N}\left(\left\langle p_{i}^{2}(t)\right\rangle-\left\langle p_{i}(t)\right\rangle^{2}\right),
$$

where $\langle\ldots\rangle$ denotes an ensemble average. This quantity can be interpreted as a dynamical analog, and plays a role similar to the physical temperature. We refer to as the $B G$-temperature, $T_{B G}$, the temperature associated with an uniform ensemble distribution in phase space. This quantity may be analytically calculated,

$$
T_{B G} \equiv \frac{1}{N} \sum_{i=1}^{N}\left[\int_{0}^{1} d p_{i} p_{i}^{2}-\left(\int_{0}^{1} d p_{i} p_{i}\right)^{2}\right],
$$

which yields $T_{B G}=1 / 12 \simeq 0.083(\forall N)$.

We studied the evolution of the dynamical temperature $T$ for typical values of the parameters as described above, focusing on the relaxation towards $T_{B G}$. We used waterbag initial conditions with homogeneous coordinate distribution $\theta_{i} \in[0,1]$, and momenta centered at $p_{0}=0.3$ with width $\delta p=0.05$. The manner in which the relaxation takes place depends strongly on the initial conditions. For example, using $0<p_{0} \ll 1$ makes the system to temporarily reach a temperature greater than $T_{B G}$, as a consequence of the periodic boundary conditions (torus $(\bmod 1))$. In general different initial conditions produce different relaxation shapes, but scaling with $N$ remains similar. We also checked inhomogeneous coordinate distributions as initial conditions $\left(\theta_{0}=0.3, \delta \theta=0.05\right)$, and obtained results qualitatively similar to those corresponding to the homogeneous case we present here. Although the temperature value in the metastable state 
is not as low as in the homogeneous case, long-lasting metastable plateaux appear (very much as in the $\alpha=0$ case of the $\alpha$-XY model, also known as Hamiltonian mean field (HMF) for initial magnetisation $M=1$ [40]). The duration of these plateaux also scales with $N$ in a qualitatively similar manner as for the homogeneous case. The particular choice of initial conditions used in this work yields a rather smooth relaxation with, for example, only one inflexion point, thus simplifying our analysis. The whole scenario is consistent with the conjecture advanced in 41.

This type of relaxation has already been reported for the particular case $\alpha=0$ [19]. A two-step process appears: firstly a stage where $T<T_{B G}$, and then a final relaxation to the predicted temperature $T_{B G}$. The initial regime varies very slowly in time yielding quasistationary (QS) states.

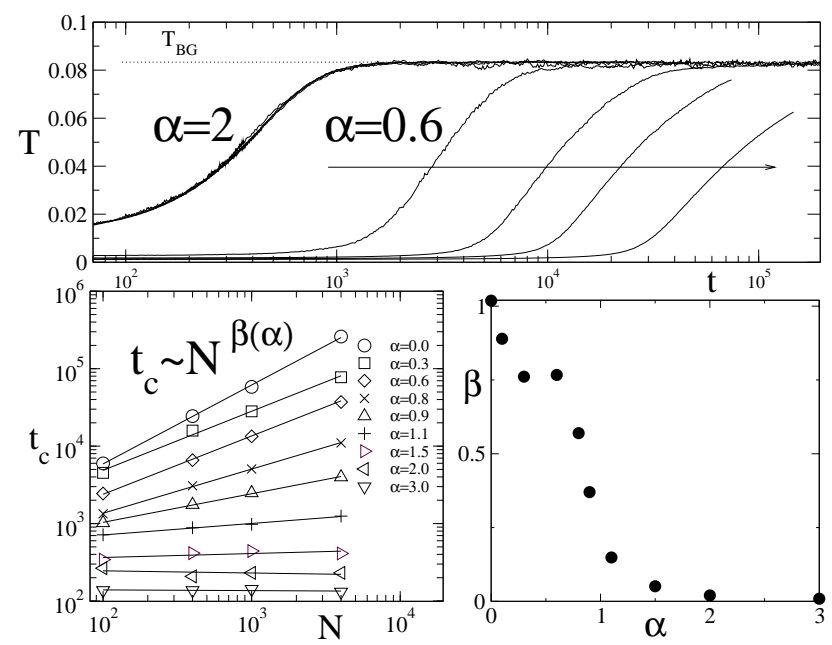

FIG. 4: Upper panel: Temperature evolution for $\alpha=2$ and $\alpha=0.6$ and four system sizes $N=100,400,1000,4000$. Initial conditions correspond to $\theta_{0}=0.5, \delta \theta=0.5, p_{0}=0.3$ and $\delta p=0.05$. Fixed constants are $a=0.05$ and $b=2$. For $\alpha=2$ the four curves coincide almost completely, all having a very fast relaxation to $T_{B G}$. For $\alpha=0.6$ the same sizes are shown, growing in the direction of the arrow. Left bottom panel: crossover time $t_{c}$ vs. $N$, showing a power-law dependence $t_{c} \sim N^{\beta(\alpha)}$ with $\beta(\alpha) \geq 0$. Right bottom panel: $\beta$ vs $\alpha$ shows that for long-range interactions the QS state lifetime diverges in the thermodynamic limit. Note that when $\alpha=0, \beta=1$, and hence $t_{c} \propto N$. Given the non-neglectable error bars due to finite size effects, the relation $\beta=1-\alpha$ is not excluded as possibly being the exact one for $0 \leq \alpha<1$ ( $\beta=0$ otherwise); more precisely, it is not unplausible that $t_{c} \propto \frac{N^{1-\alpha}-1}{1-\alpha}$ (hence, for $\left.\alpha=1, t_{c} \propto \ln N\right)$.

In Fig. 4 we show the temperature evolution for $\alpha=$ 0.6 and $\alpha=2$ for sizes $N=100,400,1000,4000$. The curves crossed by the arrow correspond to the $\alpha=0.6$ case, being the first one (from left to right) $N=100$. All four curves for $\alpha=2$ relax approximately at the same time, so it has the appearance of a single curve. For $\alpha=$ 0.6 the typical two-step relaxation is obtained. From now on, we define the crossover time $t_{c}$ from the QS state to the BG equilibrium state by means of the inflexion point, i.e. the time that corresponds to a maximum in the time derivative of $T$. The dependence of $t_{c}$ with $N$ for this choice of parameters and initial conditions is reported in the bottom left panel. The crossover time scales as $t_{c} \sim N^{\beta(\alpha)} \forall \alpha$. For $\alpha \gtrsim 1, \beta(\alpha) \approx 0$ and then $t_{c}$ remains constant (as depicted for $\alpha=2$ in Fig. 44). For $\alpha \lesssim$ $1, \beta(\alpha)>0$, i.e., $t_{c}$ diverges in the thermodynamic limit $N \rightarrow \infty$. This result indicates the inequivalence, for longrange interactions, of the orderings $t \rightarrow \infty$ and then $N \rightarrow$ $\infty$, and $N \rightarrow \infty$ and then $t \rightarrow \infty$. Consistently, these QS states become permanent (and therefore definitively relevant) when $N \rightarrow \infty$. Once again, the situation that is found coincides with that of the $\alpha$-XY model $[9]$.

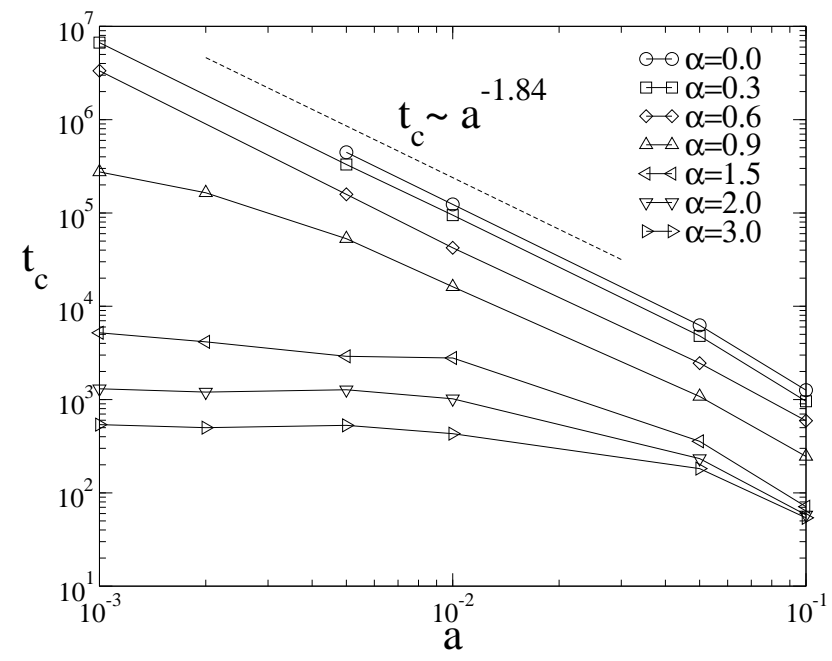

FIG. 5: Temperature dependence on $a$. Fixed constants are $N=100$ and $b=2$. Initial conditions correspond to $\theta_{0}=0.5$, $\delta \theta=0.5, p_{0}=0.3$ and $\delta p=0.05$. We averaged over 100 realisations.

Finally, we studied the dependence of $t_{c}$ with the nonlinear parameter $a$, for different values of $\alpha$, fixed coupling parameter $b=2$, and size $N=100$. In this case, as well as in the other situations analised in this work, different behaviours are reported for short- and long- range interactions. For $\alpha>1$, the value of $t_{c}$, in the limit $a \rightarrow 0$, tends to a finite value. The situation is different in the long-range case, where, for $\alpha \ll 1$, the crossover time $t_{c}$ scales as $t_{c}=d(\alpha) a^{1.84}$ being $d(\alpha)$ a nonlinear function. Preliminary calculations show that the deviation for the case $\alpha=0.9$ and $a \ll 1$ is due to finite-size effects. This scaling law implies that, for long-range interactions and vanishing nonlinear parameter $a$, the system stays in the metastable regime permanently.

Our present results for the crossover time $t_{c}$ can be approximatively summarised as follows:

$$
t_{c}(a, b, N, \alpha) \propto a^{-1.84} g_{\alpha}(b) N^{\beta(\alpha)},
$$


where $0.001 \leq a<<0.1,0 \leq b \lesssim 2, N>>1,0 \leq \alpha<1$, and $g_{\alpha}(b)$ is some function of $(\bar{b}, \alpha)$.

\section{CONCLUSIONS}

In this work we have analysed a system of $N$ symplectically and globally coupled standard maps, for both short- and long-range interactions. Our results connect with studies made in long-range Hamiltonian systems as well as with other map systems with vanishing largest Lyapunov exponent. We studied a suitably defined dynamical temperature for a region of the parameter space, and report the appearance of long-lasting quasistationary states, followed by a relaxation to the predicted equilibrium value. The relaxation time scales as $t_{c} \sim N^{\beta(\alpha)}$, with $\beta(\alpha) \approx 0$ when $\alpha \gtrsim 1$, and $\beta(\alpha)>0$ when $0 \leq \alpha \lesssim 1$, thus diverging as $N \rightarrow \infty$. This is a feature also present in the $\alpha$-XY Hamiltonian model, and constitutes a major conjecture in nonextensive statistical mechanics (NSM). Regarding the sensitivity to initial conditions, we calculated the maximum Lyapunov exponent $\lambda_{M}$ as a function of the different system parameters, namely, the size of the system $N$, the nonlinear parameter $a$ and the coupling constant $b$. We found that, as the number $N$ of maps grows, $\lambda_{M}$ vanishes as $N^{-\kappa(\alpha)}$ with $\kappa(\alpha)>0$ for $0 \leq \alpha \lesssim 1$, and $\kappa \approx 0$ for $\alpha \gtrsim 1$. The dependence of $\kappa$ with $\alpha$ is reported and compared to be the same as in the $\alpha$-XY model. We note that, even though the initial evolution of $\lambda_{M}$ depends strongly in the initial conditions (as the temperature does), this scaling does not. In fact, it coincides with that calculated with uni- form initial conditions (i.e., initial $p_{i}, \theta_{i}$ uniformly distributed in the $[0,1]$ interval) for $t \gg 1$. Our results exhibit that, in the presence of long-range interactions, the system tends to be weakly chaotic in the thermodynamic limit. This is another feature also present in the nonextensive theory that suggests its applicability. The lack of ergodicity exhibited in our model is thought to be related to a (multi)fractal constraint in the available phase-space that is enhanced in certain limits such as $N \rightarrow \infty$. This possibility would be connected to extremely low Arnold diffusion caused by the remanent of KAM tori and islands, as has been suggested for similar high-dimensional systems [42]. The similarities of the present coupled map model and the $\alpha$-XY Hamiltonian model suggest that both share features that may have a common dynamical behaviour. This fact, together with recent results obtained for low-dimensional maps, may clarify the role that NSM plays in the correct description of long-range dynamical systems.

\section{ACKNOWLEDGMENTS}

Two of us (APM, LGM) warmly thank Pablo Barberis Blostein for enlightening comments and discussions. LGM thanks Yuzuru Sato for useful remarks, and CT thanks Ezequiel G.D. Cohen and Murray Gell-Mann for deeply stimulating discussions. Financial support from CNPq (Brazilian agency), SECYT-UNC (Argentinian agency) (APM), and SI International and AFRL (USA agencies) is also acknowledged.
[1] C. Tsallis, J. Stat. Phys. 52, 479 (1988). For a recent review see M. Gell-Mann and C. Tsallis, eds., Nonextensive Entropy - Interdisciplinary Applications, (Oxford University Press, New York, 2004).

[2] L. Boltzmann, Lectures on Gas Theory, (Dover, New York, 1995);

[3] A.I. Kinchin, Mathematical Foundations of Information Theory, (Dover, New York, 1957) and Mathematical Foundations of Statistical Mechanics, (Dover, New York, 1960).

[4] D. Prato and C. Tsallis, Phys. Rev. E 60, 2398 (1999).

[5] For a regularly updated bibliography see http://tsallis.cat.cbpf.br/biblio.htm

[6] Nonextensive Statistical Mechanics and Thermodynam$i c s$, edited by S.R.A. Salinas and C. Tsallis, Braz. J. Phys. 29, Number 1 (1999); Nonextensive Statistical Mechanics and its Applications, edited by S. Abe and Y. Okamoto, Lecture Notes in Physics 560 (SpringerVerlag, Heidelberg, 2001); Non-Extensive Thermodynamics and Physical Applications, edited by G. Kaniadakis, M. Lissia, and A. Rapisarda [Physica A 305 (2002)]; Classical and Quantum Complexity and Nonextensive Thermodynamics, edited by P. Grigolini, C. Tsallis and B.J. West, Chaos, Solitons and Fractals 13, Number 3
(2002); Nonadditive entropy and nonextensive statistical mechanics, edited by M. Sugiyama, Continuum Mechanics and Thermodynamics 16 (Springer-Verlag, Heidelberg, 2004); Anomalous Distributions, Nonlinear Dynamics and Nonextensivity, edited by H. L. Swinney and C. Tsallis [Physica D 193 (2004)]; News and Expectations in Thermostatistics, edited by G. Kaniadakis and M. Lissia, Physica A 340, 1 (2004); Trends and Perspectives in Extensive and Non-Extensive Statistical Mechanics, edited by H. Herrmann, M. Barbosa and E. Curado, Physica A 344, Issue 3/4 (2004); Nonextensive Entropy - Interdisciplinary Applications, edited by M. Gell-Mann and C. Tsallis (Oxford University Press, New York, 2004); Complexity, Metastability and Nonextensivity, edited by C. Beck, G. Benedek, A. Rapisarda and C. Tsallis (World Scientific, Singapore, 2005); Nonextensive Statistical Mechanics: New Trends, New Perpectives, edited by J.P. Boon and C. Tsallis, Europhys. News 36 (6) (2005).

[7] V. Latora, M. Baranger, A. Rapisarda and C. Tsallis, Phys. Lett. A 273, 97 (2000).

[8] M. Antoni and S. Ruffo, Phys. Rev. E 52, 2361 (1995).

[9] C. Anteneodo and C. Tsallis , Phys. Rev. Lett. 80, 5313 (1998). 
[10] F.D. Nobre and C. Tsallis, Phys. Rev. E 68, 036115 (2003).

[11] F.D. Nobre and C. Tsallis, Physica A 344, 587 (2004).

[12] E.P. Borges and C. Tsallis, Physica A 305, 148 (2002).

[13] V. Latora, A. Rapisarda and S. Ruffo, Phys. Rev. Lett. 80, 692 (1998); Physica D 131, 38 (1999); Progr. Theor. Phys. Suppl. 139, 204 (2000).

[14] S.A. Cannas and F.A. Tamarit, Phys. Rev. B 54, R12661 (1996).

[15] F. Tamarit and C. Anteneodo, Phys. Rev. Lett. 84, 208 (2000).

[16] B.J.C. Cabral and C. Tsallis, Phys. Rev. E 66, 065101(R) (2002).

[17] E. Ott, Chaos in dynamical systems (Cambridge University Press, Cambridge, 1993).

[18] C. Tsallis, A. Rapisarda, V. Latora and F. Baldovin, in Dynamics and Thermodynamics of Systems with LongRange Interactions, eds. T. Dauxois, S. Ruffo, E. Arimondo and M. Wilkens Lecture Notes in Physics 602, 140 (Springer, Berlin, 2002).

[19] L.G. Moyano, A.P. Majtey and C. Tsallis, Complexity, Metastability and Nonextensivity, Proc. 31st Workshop of the International School of Solid State Physics (2026 July 2004, Erice-Italy), eds. C. Beck, G. Benedek, A. Rapisarda and C. Tsallis (World Scientific, Singapore, 2005), p. 123.

[20] F. Baldovin, L.G. Moyano, A.P. Majtey, A. Robledo and C. Tsallis, Physica A 340, 205 (2004).

[21] F. Baldovin and A. Robledo, Phys. Rev. E 66, 045104(R) (2002); F. Baldovin and A. Robledo, Phys. Rev. E 69, 045202(R) (2004); F. Baldovin and A. Robledo, Europhys. Lett. 60, 518 (2002). See also E. Mayoral and A. Robledo, Physica A 340, 219 (2004), E. Mayoral and A. Robledo, Phys. Rev. E 72, 026209 (2005), and H. Hernandez-Saldana and A. Robledo, Phys. Rev. E (2006), in press cond-mat/0507624.

[22] The q-exponential is defined as $\exp _{q}(x) \equiv[1+(1-$ q) $x]^{1 / 1-q}$ and its inverse is the q-logarithm, $\ln _{q}(x) \equiv$ $\left(x^{1-q}-1\right) /(1-q)$. The usual exponential and logarithm are recovered when $q \rightarrow 1$.

[23] A. Robledo, Phys. Lett. A 328, 467 (2004); F. Baldovin and A. Robledo, Phys. Rev. E 72, 066213 (2005).

[24] F. A. B. F. de Moura, U. Tirnakli and M. L. Lyra, Phys. Rev. E 62, 6361 (2000).

[25] U. Tirnakli, C. Tsallis and M.L. Lyra, Eur. Phys. J. B 11, 309 (1999); U. Tirnakli, C. Tsallis and M.L. Lyra, Phys.
Rev. E 65, 036207 (2002). U. Tirnakli, Phys. Rev. E 66, 066212 (2002); U. Tirnakli, Physica A 305, 119 (2002); E. P. Borges and U. Tirnakli, Physica D 193, 148 (2004); E. P. Borges and U. Tirnakli, Physica A 340, 227 (2004);

[26] G. Casati, C. Tsallis and F. Baldovin, Europhys. Lett. 72, 355-361 (2005).

[27] V. Latora and M. Baranger, Phys. Rev. Lett. 82, 520 (1999).

[28] F. Baldovin, E. Brigatti and C. Tsallis, Phys. Lett. A 320, 254 (2004).

[29] M. Kac, G. Uhlenbeck and P.C. Hemmer, J. Math. Phys. 4, 216 (1963).

[30] V. Ahlers, R. Zillmer, and A. Pikovsky, Phys. Rev. E 63, 036213 (2001).

[31] T. Konishi and K. Kaneko, J. Phys. A 25, 6283 (1992); K. Kaneko and T. Konishi, Physica D 71, 146 (1994).

[32] M.-C. Firpo and S. Ruffo, J. Phys. A 34, L511 (2001); D. Moroni, Dynamics and thermodynamics of a variablerange interacting $X Y$ model, M. Sc. Thesis, University La Sapienza, Rome, Italy (2000).

[33] G. Benettin, L. Galgani, A. Giorgilli and J.M. Strelcyn, Meccanica 15, 21 (1980).

[34] C. Anteneodo, Phys Rev E 69, 016207 (2004).

[35] This is a consequence of the Oseledec theorem that states that, assuming ergodicity, (almost) any exponential growth tends to an asymptotic value $\lambda_{0}$, independently of the orbit and for (almost) all intial conditions. See for example, V.I. Oseledec, Trans. Mosc. Math. Soc. 19, 197 (1968), J.-P. Eckmann and D. Ruelle, Rev. Mod. Phys. 57, 617 (1985).

[36] G.M. Zaslavsky, R.Z. Sagdeev, D.A. Usikov and A.A. Chernikov, Weak chaos and quasi-regular patterns (Cambridge University Press, Cambridge, 1991).

[37] B.V. Chirikov, Phys. Rep. 52, 263 (1979).

[38] M.-C. Firpo, Phys. Rev. E 57, 6599 (1998).

[39] C. Anteneodo and R.O. Vallejos, Phys. Rev. E 65, 016210 (2002); R.O. Vallejos and C. Anteneodo, Phys. Rev. E 66, 021110 (2002); R.O. Vallejos and C. Anteneodo, Physica A 340, 178 (2004).

[40] A. Pluchino, V. Latora, A. Rapisarda, Physica A 338, 60 (2004).

[41] C. Tsallis, Braz. J. Phys. 29, 1 (1999).

[42] M. Falcioni, U. Marini Bettolo Marconi and A. Vulpiani, Phys. Rev. A 44, 2263 (1991). 